\title{
Philosophiques
}

\section{La contribution silencieuse de Husserl à l'herméneutique}

\section{Jean Grondin}

Volume 20, numéro 2, automne 1993

Perspectives sur la phénoménologie et l’intentionnalité

URI : https://id.erudit.org/iderudit/027232ar

DOI : https://doi.org/10.7202/027232ar

Aller au sommaire du numéro

Éditeur(s)

Société de philosophie du Québec

ISSN

0316-2923 (imprimé)

1492-1391 (numérique)

Découvrir la revue

Citer cet article

Grondin, J. (1993). La contribution silencieuse de Husserl à l'herméneutique. Philosophiques, 20(2), 383-398. https://doi.org/10.7202/027232ar d'utilisation que vous pouvez consulter en ligne.

https://apropos.erudit.org/fr/usagers/politique-dutilisation/ 

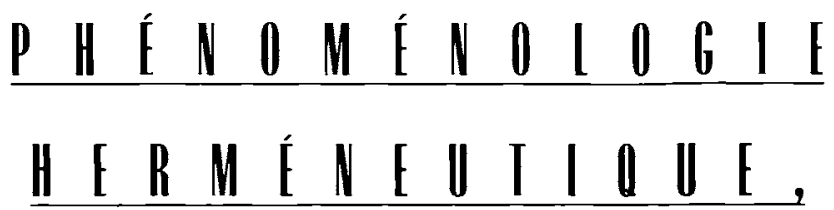

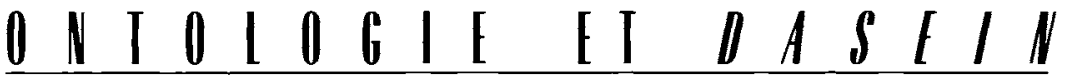

\section{LA CONIRIBUTION SILENCIEUSE DE HUSSERL À L' HERMÉNEUTIOUL}

\section{par \\ Jean Grondin}

Si Husserl a contribué à l'herméneutique, c'est bien malgré lui. Husserl était en effet doublement allergique à la pensée qu'on peut qualifier d'herméneutique. Il ne tolérait pas son historicisme, comme en témoigne son débat avec Dilthey en IgII, mais aussi le débat plus secret, donc amer, qu'il a mené avec l'herméneutique de Heidegger. Husserl ne reconnaissait pas non plus une importance de premier plan à l'idée d'interprétation. En termes très simples, mais éminemment dignes de Husserl, ce qui l'intéressait, ce n'étaient pas les interprétations des phénomènes, mais les phénomènes eux-mêmes. Dans une étude remarquable ${ }^{\mathrm{I}}$, Paul Ricoeur a cependant attiré l'attention sur l'emploi par Husserl de termes comme ceux de Deutung et d'Auslegung (interprétation), qui montreraient que son allergie herméneutique n'était peut-être pas totale. Mais, pour Husserl, s'il faut « interpréter » certains phénomènes, s'il faut tenir compte des variations interprétatives [Abschattungen] des choses, c'est justement pour mieux parvenir à leur essence. L'intuition des essences n'a pas un sens bien sorcier chez Husserl. Au fil des interprétations

I. P. Ricoeur, «Phénoménologie et herméneutique », dans son recueil Du texte à laction. Essais d'herméneutique II. Paris, Seuil, 1986, p. 39-73. 
intentionnelles, on finit toujours par voir ce qui est essentiel à un phénomène. Retourner aux choses elles-mêmes, cela veut dire qu'il faut savoir se défaire de l'emprise déformante des théories et des interprétations unilatérales des phénomènes, pour retourner à l'essentiel, à ce qui se maintient à travers toutes les interprétations. D'où l'utilité de multiplier les « interprétations » pour s'assurer que l'essentiel ait été bien cerné.

Il est un peu ironique de constater qu'une philosophie qui cherchait à libérer la philosophie des interprétations, des théories et des livres ait donné naissance à une philosophie « continentale » dont l'orientation historique et livresque soit aussi prononcée. Comme l'écrit justement Gadamer :

Chaque phénoménologue avait sa propre conception au sujet de ce qu'était réellement la phénoménologie. Une seule chose était certaine : c'est qu'on ne pouvait apprendre la méthode phénoménologique à partir des livres ${ }^{2}$.

Il est bien connu que Husserl a lui-même assez peu publié. Ce qui l'intéressait, c'était la recherche plus que la publication philosophique. Il n'a d'ailleurs publié qu'un seul des trois tomes de ses Ideen, lequel parut d'abord sous forme d'« article »dans le premier volume des Annales pour la philosophie et la recherche phénoménologique. Et ce tome ne proposait que « des » idées en vue d'« une » [Ideen zu einer...] phénoménologie pure et d'une philosophie phénoménologique. Rien n'indique donc qu'il se soit agi pour Husserl d'une fondation définitive ou de la seule qui soit possible. Par la suite, Husserl n'a presque plus publié. Il estimait d'ailleurs que ses élèves, formés à l'évidence presque géométrique de la phénoménologie, étaient capables d'accomplir aussi bien que lui le travail technique d'écriture et de publication. C'est ce qui explique, par exemple, pourquoi il a mis tant de temps à s'apercevoir des différences qui le séparaient de Heidegger. Persuadé que ce dernier était acquis à la cause de la phénoménologie, il ne s'est pas donné la peine de prendre connaissance de ses écrits, faisant même

2. H.G. Gadamer, « Die phänomenologische Bewegung », dans ses Gesammelte Werke, t. III, Tübingen, J. C. B. Mohr (Paul Siebeck), 1987, I16. « Jeder Phänomenologe hatte seine eigene Meinung über das, was Phänomenologie eigentlich sei. Nur eins galt als gewi $\beta$, da $\beta$ man die phänomenologische Arbeitsweise nicht aus Büchern lernen könne ». Cf. déjà M. Heidegger en 1924, Platon: Sophistes, Gesamtausgabe ig, Francfort, Klostermann, 1992, p. 9 : «L'introduction à la phénoménologie ne se fait pas par la lecture de la littérature phénoménologique. » 
paraître Être et temps dans ses propres Annales sans en avoir lu une seule ligne ${ }^{3}$.

Les élèves de Husserl ont aussi hérité de sa réserve, toute platonicienne, vis-à-vis de la publication. Ainsi que l'a noté Gadamer, la production littéraire de Husserl et de la première génération de phénoménologues demeura assez modeste: onze tomes du Jahrbuch parus sur deux décennies et à peu près rien dans les autres organes de publication ${ }^{4}$. Il est bien connu que c'est à la suite des pressions d'un ministre que Heidegger s'est résolu à sortir son seul livre véritable, et qui n'était lui-même qu'un programme, Être et temps, qui parut aussi dans un tome du Jahrbuch. Quant à Gadamer, ce sont ses étudiants qui l'ont poussé, alors qu'il était déjà âgé de soixante ans, à publier Vérité et méthode (Ig6o), son premier livre depuis sa dissertation universitaire de ig29.

L'essentiel étant de penser à partir des choses elles-mêmes, Husserl fit preuve d'une très grande libéralité en laissant à ses élèves, sans doute à leur instigation, le soin de publier quelquesuns de ses cours. M. Heidegger édita les leçons sur la conscience intime du temps et Ludwig Landgrebe colligea plusieurs cours et manuscrits qui servirent de base à la rédaction de Logique formelle et transcendantale (qui parut dans le Jahrbuch en $\mathbf{I g}^{2}$ f $^{5}$. Quelques années plus tard, Landgrebe, suivant l'autorisation [Vollmacht] de Husserl, se fonda aussi bien sur des cours, des manuscrits que des conversations orales avec son maître lorsqu'il assuma entièrement la rédaction de Expérience et jugement (1939) ${ }^{6}$. Husserl confia aussi à son jeune élève Eugen Fink la tâche de rédiger une « sixième » méditation cartésienne qui viendrait s'ajouter aux quatre conférences qu'il avait prononcées

3. Cf. O. PUggeler, « Die Krise des phänomenologischen Philosophiebegriffs (rgzg) », dans Phänomenologie im Widerstreit, édité par Ch. Jamme et O. Pöggeler, Francfort, Suhrkamp, 1989, p. $255^{-276}$. Au reste, Heidegger affectait la même indifférence à l'endroit des publications de son « maître »: lorsqu'il a voulu reconnaître sa dette envers Husserl à la fin de l'Introduction à Être et temps, Heidegger s'est contenté de faire allusion à « sa direction personnelle exigeante » et à la " générosité avec laquelle il nous a ouvert l'accès à ses recherches inédites » (§ 7, trad. E. Martineau, Paris, Authentica, 1985, p. 49).

4. H.-G. Gadamer, op. cit. p. 116 .

5. Formale und transzendentale Logik, Husserliana XVII, P. xxII, 2I.

6. L. Landgrebe, Vorwort des Herausgebers : E. Husserl, Erfahrung und Urteil, PTague, Academia, 1939, V-XI. 
à Paris. Cette sixième méditation de Fink (« et »de Husserl) ne fut publiée qu'en 1988 , dans la collection des Husserliana ${ }^{7}$.

Les dernières publications de Husserl, les Méditations cartésiennes et la Krisis, sont nées de conférences. Même s'il fallut attendre l'édition posthume des Husserliana pour en obtenir le texte intégral, Husserl, pour une fois, semble avoir prêté une attention toute spéciale à leur publication. Confronté au succès de Sein und Zeit, Husserl aura été cruellement amené à prendre conscience de l'efficace de l'écrit en philosophie. Souhaitant contrer l'influence « néfaste » qu'exerçait selon lui l'ouvrage de Heidegger, Husserl espérait, en effet, que de nouveaux écrits puissent conférer une nouvelle voix et un nouveau poids à la pensée authentiquement phénoménologique ${ }^{8}$. Le détour de l'écrit était redevenu nécessaire pour réitérer la rigueur du retour aux choses elles-mêmes. La tragédie politique de l'Allemagne a cependant anéanti les projets et les espoirs de Husserl. C'est à Belgrade que dut paraître la Krisis en 1936. Pendant de longues décennies, la voix de Husserl demeura douloureusement inaudible en phénoménologie.

Il reste néanmoins toujours possible d'entendre le sens proprement herméneutique de l'appel à un retour aux choses elles-mêmes, par delà l'écrit, les formules et les théories : von den bloßen Worten [... zu den Sachen Selbst - « des simples mots [...] vers les choses elles-mêmes ${ }^{9}$. Il est redevenu courant en philosophie,

7. E. Fink, VI.Cartesianische Meditation, éd. par H. Ebeling, J. Holl et G. van Kerckhoven (2 tomes), Dordrecht, Kluwer Academic Publishers, I988 (Husserliana-Dokumente Band II / I et II / 2). Le texte allemand des quatre conférences de Husserl ne parut qu'en ig5o dans l'édition des Husserliana.

8. Cest l'hypothèse très vraisemblable que propose $\mathrm{K}$. Schumann, « $\mathrm{Zu}$ Heideggers Spiegel-Gespräch über Husserl », dans Zeitschrift für philosophische Forschung. 32, 1978, p. 603. Schumann cite à ce propos une lettre de Husserl à Ingarden Idu 2 décembre 1929). Après que ce demier eut évoqué les résultats de son étude de Heidegger, Husserl répondit laconiquement : «j'accorde donc d'autant plus de poids au déploiement intégral de l'édition allemande des Méditations cartésiennes ». Cf. aussi H. G. Gadamer, « Europa und die Oikumene», dans Europa und die Philosophie, hrsg. von H. H. Gander. Francfort, V. Klostermann, I993, p. 68 : « Nicht zufällig hat Husserl später in der Abgrenzung gegen Heidegger und andere seine "Cartesianische Meditationen" verfaßt » (« ce n'est pas un hasard si le demier Husserl a publié des "Méditations cartésiennes" pour se distinguer de Heidegger $\gg)$.

9. Logische Untersuchungen, Husserliana $\mathrm{XIX} \mathrm{/} \mathrm{I,} \mathrm{§} \mathrm{2,} \mathrm{p.} \mathrm{Io.} \mathrm{Pour} \mathrm{la} \mathrm{juste} \mathrm{intelligence} \mathrm{de}$ ce mot d'ordre, qu' on peut opposer au « ad fontes » des humanistes et au « retour à Kant » des néo-kantiens, cf. H. Spiegelberg. The Phenomenological Movement. $A$ Historical Introduction, $3^{\mathrm{e}}$ édition revue et augmentée, Martinus Nijhoff, La Haye / 
comme il l'était déjà à l'époque de Husserl, de discuter presque exclusivement de théories, d'arguments ou de propositions que l'on cherche à faire dériver de propositions supposément plus fondamentales. Aucune philosophie, bien sûr, n'échappe au cycle de l'argumentation, de la mise en théorie et de la contextualisation historique, mais par-delà le déluge des propositions, il y a toujours un sens à entendre et des choses à voir, et qu'il faut voir si l'on veut en parler. Les élèves les plus herméneutes de Husserl, c'est-à-dire Heidegger et Gadamer, ont tout naturellement conçu le phénomène de la compréhension selon le modèle que Husserl leur avait proposé. Comprendre, c'est remonter du dit au sens qui l'anime, du discours extérieur à la question qui le motive. Toute la polémique de Heidegger contre le jargon du « on » n'est en fait qu'une application herméneutique de l'injonction phénoménologique du retour aux choses elles-mêmes. Les lieux communs du « on » nous déchargent en effet d'une vision directe des choses, celle que nous sommes pourtant en mesure d'acquérir par nousmêmes, s'il est vrai que nous sommes, en principe, des «Da-sein », des êtres susceptibles d'être « là », c'est-à-dire directement auprès des choses, lorsque tombent les décisions fondamentales quant à notre existence. L'appel à l'authenticité qui constitue le Dasein est celui d'un retour aux choses, d'une confrontation directe avec soi.

Toute la critique du discours propositionnel dans Sein und Zeit s'effectue sous l'impératif d'un retour herméneutique à l'ordre antéprédicatif qui donne corps au discours réel ${ }^{10}$. Quelles sont les choses qui veulent se faire entendre derrière ou « dans »le discours ? Gadamer s'inspire à la fois de Husserl et de Heidegger lorsqu'il cherche à comprendre le langage à partir du dialogue, suivant le fil conducteur d'une « logique » de la question et de la réponse. On ne comprend le discours - d'autrui, d'un texte - que si l'on sait à quelle question il cherche à répondre. Tout énoncé est réponse à une question. Or, si l'on veut entrer dans l'horizon de

Boston / Londres, 1982, p. I09. Cf. aussi G. Heffernan, Am Anfang war die Logik. Hermeneutische A bhandlungen zum Ansatz der Formalen und tranzendentalen Logikvon Edmund Husserl, Verlag B. R. Grüner, Amsterdam, Ig88, p. 13. qui souligne la portée herméneutique de la remontée husserlienne « des mots vers les choses ellesmèmes ». Comme le rappelle Heffernan, c'est Heidegger qui a imposé à la phénoménologie la formule plus lapidaire « vers les choses elles-mêmes ».

Io. Sur la lutte de Heidegger avec le régime propositionnel, of. notre étude sur l'intelligence herméneutique du langage, dans Lhorizon herméneutique de la pensée contemporaine, Paris, Vrin, 1993. p. 253-269. 
cette question (et la notion d'horizon, si importante pour l'herméneutique, vient directement de Husserl), force est de retourner aux choses elles-mêmes, en amont du discours. Paul Ricoeur a donc parfaitement raison d'apercevoir dans la thèse du caractère «secondaire » de l'ordre linguistique « la plus importante présupposition phénoménologique de l'herméneutique $»^{\mathrm{II}}$. Le discours propositionnel ne dit jamais tout ce qui est essentiel à sa pénétration. Pour en saisir le sens, il faut retourner aux choses elles-mêmes, s'introduire dans le dialogue dont procède l'énoncé. C'est qu'il y a toujours un décalage entre la chose vue et le langage qui l'exprime, entre la chose au singulier et les mots pluriels qui la balbutient. L'abîme qui se creuse entre le discours extérieur et l'intériorité qu'il cherche à faire entendre est éminemment herméneutique ${ }^{\mathrm{I} 2}$. Si la phénoménologie reste une urgence pour la philosophie, c'est qu'il est souvent commode d'en rester au seul niveau des théories et des discours, sans se soucier des choses elles-mêmes. Ce qui importe dans le régime autonome des théories, c'est plus la cohérence interne que ladéquation du discours. Pour la phénoménologie, la vérité reste affaire d'adéquation. Il est donc essentiel de lutter contre ce que Heidegger, dans le cours de 1924 sur le Sophiste ${ }^{\mathrm{I} 3}$, a superbement nommé « la déchéance dans les logoi », la propension du Dasein à s'abandonner à l'assurance des opinions reçues.

Le « retour » aux choses obéit lui-même à la logique de la question et de la réponse. Il ne tombe pas du ciel. On ne l'a pas toujours bien vu, mais le Zurück zu den Sachen selbst, le « re-tour » aux choses tourne le dos (littéralement : le Rücken) à quelque chose. Le retour présuppose un détour, c'est-à-dire un détournement des choses à la faveur du règne des seuls discours. Sur ce point, la solidarité de Husserl et de Heidegger est entière, même si leur point de départ apparaît un peu différent. En effet, lorsque Husserl proclame son « retour » aux choses elles-mêmes, il veut avant tout tourner le dos au royaume prétendument autonome de la «théorisation » scientifique qui n'a pas fait l'objet d'une légitimation directe à même les choses elles-mêmes, à même le

II. Op.cit., 60.

12. Cf. notre ouvrage sur L'universalité de lherméneutique, Paris, P.U.F., coll. Epiméthée, I993.

13. M. Heidegger, Platon:Sophistes, Gesamtausgabe ig, Francfort, Klostermann, I992, p. Ig, 27,47 et passim. 
monde vécu, que doit pourtant servir la science. Depuis les Recherches logiques jusqu'à la Krisis, cette critique de l'abstraction au profit de l'évidence des choses elles-mêmes traverse comme un fil rouge toute l'œuvre de Husserl. Issu d'une science aussi apodictique que la géométrie, où la vision des essences reste, si l'on peut dire, l'essentiel, il n'a jamais compris pourquoi ses collègues philosophes s'appuyaient, avec autant de constance que de cécité, sur des théories préalables, qu'il s'agisse des théories scientifiques elles-mêmes, dont la philosophie prétendrait être la « métathéorie », ou des « classiques » dans la pratique de l'histoire des problèmes philosophiques. Contre l'épistémologie, mais aussi contre l'histoire de la philosophie érigée en fin en soi, Husserl martèle la nécessité d'un débat qui porte directement sur les choses elles-mêmes. On a ainsi tort de critiquer le manque d'érudition philosophique ou historique d'un penseur comme Husserl. Naivement ou courageusement, Husserl estime que l'essentiel n'est pas là. Ce qui compte vraiment, c'est de parler des choses elles-mêmes, ce qui exige cependant une conversion totale du regard philosophique, une mise entre parenthèses de l'attitude naturelle, bref, une « réduction » phénoménologique. Nous y reviendrons.

Heidegger, quant à lui, imprime un sens plus existential à l'injonction d'un retour aux choses elles-mêmes. Son «zurück zu den Sachen selbst » ne se limite pas au phénomène de la « théorie abstraite » qui avait tant irrité un penseur comme Husserl, dont la formation était d'abord mathématique. Chez Heidegger, le retour aux choses est d'abord commandé par le phénomène de l'« inauthenticité » ou du bavardage. C'est qu'il s'agit pour Heidegger d'un manquement essentiel du Dasein à soi-même. Être un Dasein, comme on l'a noté, c'est justement être capable d'être « là », c'est-à-dire de s'ouvrir les yeux et de voir par soi-même. Or, le plus souvent, croit constater Heidegger, le Dasein choisit plutôt de ne pas voir par lui-même et de s'en remettre à l'autorité avérée et omnipotente du bavardage [Gerede]. Le retour heideggérien aux choses elles-mêmes s'élève contre cette hégémonie des discours qui n'ont d'autre créance que celle d'avoir été répétés ou communément acceptés (c'est d'ailleurs l'une des limites de l'éthique de la discussion). Toute la problématique du cercle herméneutique reste dominée par cette urgence phénoménologique, même si l'on a souvent pensé que Heidegger s'écartait de la phénoménologie de son maître en lui insufflant un tournant herméneutique. C'est 
que pour Heidegger la tâche constante et ultime de l'interprétation

[...] reste non pas de se laisser pré-donner la pré-acquisition, la prévision et l'anti-cipation par des « intuitions » ou des concepts populaires IVolksbegriffel, mais, en les élaborant, d'assurer toujours son thème scientifique à partir des choses mêmes ${ }^{\mathrm{I4}}$.

Le retour heideggérien aux choses elles-mêmes tourne donc le dos à l'inauthenticité qui gangrène le Dasein lorsqu'il cède IVerfallenl à la facilité des lieux communs et des évidences du « on » au lieu de s'ouvrir les yeux et d'assumer pour lui-même les décisions fondamentales qui concernent son orientation dans l'existence. D'abord conçue comme réponse à la primauté des théories mal assurees, le retour aux choses elles-mêmes est devenu une réponse à l'inauthenticité du Dasein. Mais d'une phénoménologie à l'autre, la continuité tombe sous le sens puisque l'exigence d'authenticité se trouvait déjà inscrite dans l'appel husserlien à un retour aux choses elles-mêmes, lequel présupposait que les théories abstraites passaient à côté de l'essentiel ${ }^{15}$.

L'injonction cardinale de la phénoménologie tient donc à cette exigence de la vision, par-delà les signes et les écrits. La maîtrise d'un langage ou d'un jargon ne signifie jamais que la chose elle-même ait été maîtrisée. L'intelligence, en tout, se mesure à ce qu'il y a derrière le langage, à ce qui a été vu derrière les mots qui ont été proférés. Voilà ce qui a d'abord fasciné Heidegger dans sa première appropriation de la phénoménologie ${ }^{\mathrm{I}}$. Mais Husserl avait été le premier à y reconnaître le principe de tous les principes dans ses Ideen : toute intuition originairement donatrice est une

14. M. Heidegger, Sein und Zeit, $\S 32$, p. 153 ; Être et temps, trad. E. Martineau, Paris, Authentica, 1985, p. 124. On notera à ce propos que la problématique herméneutique de la compréhension et de l'interprétation dans $S Z$ ( $\$ 28$ à 34) se trouve encadrée par l'analyse du « on » (\$27) et celle du bavardage (\$34). C'est que la nécessité d'une interpretation explicitante à partir des choses elles-mêmes vise à combattre ce que Heidegger appelle la « dictature du on » (SZ, p. 126 ; tr. Martineau, p. 108) et dont le bavardage constitue l'articulation la plus assourdissante.

15. Sur le motif récurrent de l'inauthenticité et de son corollaire, le déclin, chez Husserl, $c f$ a titre exemplaire Formale und transzendentale Logik, Husserliana XVII, p. 32 : « Nous parlions d'une connaissance authentique, d'une science authentique, d'une méthode authentique. Les idées logiques sont d'emblée des idées d"authenticité" (Echtheit). L'authentique, c'est ce vers quoi tend en demière instance la raison, même dans son mode déclinant/Verfallsmodus/ de déraison » (tous les soulignés sont de Husserl).

16. Cf. ses « maximes fondamentales de la phénoménologie »dans le cours de 1919 / D: M. Heidegger, Grundprobleme der Phänomenologie, Gesamtausgabe, Bd. 58 , Franfkurt, Klostermann, 1993, P. 219 
source originaire de connaissance, etc. On ne cite cependant pas toujours les cinq petits mots qui précèdent le principe des principes dans les Ideen au début du § 24 : « Mais [c'en estl assez des théories farfelues! Doch genug derverkehrten Theorien $\gg{ }^{17}$. Ce doch au tout début d'un chapitre est une véritable parataxe. Son audace fonde toute la phénoménologie. Ce que le principe des principes a de remarquable, c'est qu'il n'explique rien. Rien ne « découle » logiquement de ce principe. Il ne comporte ni corollaires, ni scolies. $S$ 'il n'explique rien, ce principe justifie tout. Tout ce qui se dit doit pouvoir être reconduit à une intuition. L'essentiel est de voir ce dont on parle.

C'est sur cette exigence herméneutique de probité que repose selon Husserl tout l'exercice de la philosophie en tant que science qui se veut première. Le plus simplement du monde, la philosophie doit partir des choses telles qu'elles se donnent dans l'intuition. D'où l'urgence de donner congé aux théories qui courent et qui ne se fondent pas directement dans une phénoménalité qui se laisse voir, donc partager. Il ne suffit plus pour un phénoménologue d'enchaîner les arguments et les formules. A chaque étape de sa « recherche » (et le terme de Forschung comporte ici son sens plein, celui d'une réelle exploration des phénomènes), la phénoménologie doit s'assurer d'une légitimation directe à même les choses. Dans un appel qui devint plus révolutionnaire qu'il n'osa lui-même l'imaginer, puisqu'il finit par se retourner contre certaines admissions de sa phénoménologie, Husserl invite la pensée rigoureusement philosophique à se garder de toute construction « métaphysique » pour s'en tenir à ce que l'intuition «donne ». Dans la mesure où elle cherche à s'affranchir de ce qui ne se laisse pas directement donner dans l'intuition, la phénoménologie aura préparé le dépassement de la métaphysique entrepris par l'herméneutique plus récente ${ }^{\mathrm{I}}$.

L'injonction d'un retour aux choses elles-mêmes se trouve cependant souvent taxée de naïveté ou de trivialité. Quelle

\footnotetext{
17. Husserliana III, P. 5 I.

18. On comprend donc que la phénoménologie soit. d'un point de vue méthodologique, rebelle à la théologie (à tout le moins, celle qui provient de la métaphysique). Cf. à ce propos l'essai polémique de D. Janicaud sur Le toumant théologique de la phénoménologie française, Paris, L'éclat, ıg9 qui a le double mérite de trouver un dénominateur commun à la phénoménologie française la plus récente et de confirmer l'actualité critique de la phénoménologie.
} 
théorie, en effet, ne prétend pas parler au nom des phénomènes? Or l'injonction critique de Husserl se double d'une critique, ou, plus exactement, d'une suspension de la naïveté propre à l'attitude naturelle. Au nom des droits les plus élémentaires de l'intuition, Husserl nous invite en effet à pratiquer une réduction phénoménologique, à une épochè de l'attitude la plus naturelle qui consiste à s'en remettre à l'évidence du monde «transcendant ». Le terme de « réduction » comporte une ambiguité essentielle. Il paraît d'abord posséder une signification un peu positiviste. C'est ainsi que l'on parle parfois d'une interprétation « réductrice » lorsqu'elle simplifie le réel ou qu'elle le ramène à un seul de ses aspects : ceci se réduit à cela. Or la « réduction » husserlienne a plutôt le sens d'une « re-direction » du regard, suivant l'étymologie de la re-ductio, que l'on pourrait rendre en parlant d'une « reduction » sans accent aigu. L'essentiel de la réduction réside pour Husserl dans une conversion de la vision. Il s'agit encore une fois d'arracher le regard à l'emprise des évidences mondaines qui circulent, pour le re-conduire [re-ductiol à l'évidence première, c'est-àdire, pour Husserl, au monde tel qu'il se donne. Or ce monde, c'est bien connu et l'on y salue généralement l'un des grands acquis de la phénoménologie, est d'abord donné comme phénomène intentionnel, si bien que la re-ductio phénoménologique donnera lieu à une Forschung, une exploration, de l'intentionnalité.

Deux moments herméneutiques méritent d'être soulignés dans cette idée de re-ductio. Tout d'abord l'idée que l'accès aux choses essentielles procède justement d'une re-conduction du regard. L'intelligence herméneutique doit partir des signes qui sont donnés, mais elle doit aussi savoir s'en délivrer afin de se rediriger vers l'intention qui anime ce qui a été dit. Comprendre, c'est effectuer une « réduction » du regard, savoir prendre une distance envers ce qui se raconte, afin de percer vers le sens, sur ce qui veut être compris. Mais la réduction découvre aussi l'infinité de la tâche herméneutique. Si le sens vers lequel l'intelligence herméneutique se tourne relève d'une intentionnalité, d'une « visée » de sens, c'est dans cette visée qu'il faut pénétrer si l'on est phénoménologue. On mesure toutefois la difficulté de l'entreprise : il s'agit de viser une visée, de s'engager intentionnellement dans l'intentionnalité. Certes, l'intentionnalité semble constituer une donnée ultime pour la phénoménologie (dans ses versions scolaires à tout le moins), mais cette « chose » fondamentale, ou plus fondamentale que toutes les données qui en 
jaillissent (le monde, la chose, etc.), est embrasée d'une motion centrifuge : l'intentionnalité est nécessairement tension hors de soi, vers le sens. C'est ici que se consomme l'union de la phénoménologie et de l'herméneutique : le retour à la chose elle-même qu'est l'intentionnalité se trouve devant une donnée qui est ellemême visée de sens. Ce qu'il faut « voir » ici a de soi-même le caractère d'une vision ou d'une visée. Il faut voir la vision ellemême, pratiquer une espèce de noesis noeseos (analogie qu'atteste, bien sûr, le lexique de la noèse chez Husserl lui-même). On aurait cependant tort d'assimiler cette vision de la vision à une pure « égologie », même si Husserl y a parfois été re-conduit, car la visée de sens vise en fait l'hors de soi. En termes apparemment techniques, mais que nous espérons intelligibles : la noèse de l'ego dans l'intentionnalité est d'emblée, en tant que visée, ouverture sur l'autre, elle n'est pas visée de soi. Il y a bien sûr « corrélation » entre la visée et ce qui est visé, mais le noème ne se réduit pas à la noèse. Il y a toujours tension entre ce que la conscience vise et le sens visé lui-même, lequel outrepasse la conscience égologique. Cette tension entre le sens visé et le sens lui-même mérite d'être appelée herméneutique.

La réduction husserlienne, quelle que soit la forme précise qu'elle en vienne à prendre let les Husserliana nous en révèlent toujours de nouvelles) ${ }^{19}$, s'institue à chaque fois comme reductio vers la constitution du sens, la Sinnkonstitution. Comme celui de réduction, le terme de constitution a souvent prêté à malentendu. On a souvent cru que le sujet transcendental était un peu le «créateur »du sens ${ }^{20}$. Pareille généalogie transcendantale est toutefois étrangère à la phénoménologie, bien que la terminologie de Husserl rappelle parfois l'idéalisme. L'idée phénoménologique de constitution veut seulement souligner que le « sujet » de l'intention co-constitue toujours le sens, puisqu'il est toujours « là » lorsque le sens advient, comme Heidegger aura voulu le souligner avec son heureux concept de «Da-sein ».

I9. Dès les Ideen, l'important chapitre IV consacré à cette thèmatique parlait toujours des phänomenologischen Reduktionen au pluriel. Cf. Ideen, $\S 56$ à $6 \mathbf{2}$.

20. Cf. l'utile mise au point de F. Dastur dans sa présentation de Husserl dans La philosophie allemande de Kant à Heidegger, sous la direction de D. Folscheid, Paris. P.U.F., coll. Premier Cycle, 1993, p. 273 : « Dans l'attitude phénoménologique, la conscience fait lexpérience de son pouvoir constituant, ce qui ne veut pourtant pas dire qu'elle est pourvue d'une puissance créatrice qui légalerait à la pensée divine. Husserl l'a toujours clairement souligné, constitution ne veut pas dire pour lui création ex nihilo, mais donation de sens. » 
L'un des grands mérites herméneutiques de Husserl est de ne jamais réduire le sens à la visée intentionnelle du sujet. Le sujet ne fait que co-constituer le sens, que naître avec lui (suivant le « connaître » de Péguy) : l'ego demeure en dialogue constant avec un sens qu'il ne maîtrise jamais totalement. D'où l'importance pour la phénoménologie husserlienne d'une exploration des « couches » de l'intentionnalité ou des différentes étapes de la constitution de sens qui échappent à la conscience immédiate et naturelle. Gadamer en a bien retenu la motivation :

La constitution ne signifie rien d'autre que le «mouvement de reconstruction » qui suit l'accomplissement de la réduction. [...] La construction à partir des prestations de la subjectivité ne désigne pas un engendrement effectif de quoi que ce soit, mais seulement le chemin de la compréhension de tout ce qui peut valoir comme sens $^{2 I}$.

Le problème de la constitution chez Husserl veut seulement reconstituer l'horizon de sens de ce qui est visé par la conscience intentionnelle.

En orientant la phénoménologie vers le phénomène de la constitution (ou donation) de sens, Husserl ouvre donc la voie à l'herméneutique, mais aussi à une mise en cause par l'herméneutique de certains présupposés non phénoménologiques de la phénoménologie ${ }^{22}$. Mais cette déconstruction aura aussi été rendue possible par Husserl lui-même. Il avait été le premier à inciter ses étudiants à se méfier des théories dont les assises phénoménologiques n'étaient pas établies. Il les invitait ainsi à une critique permanente des présupposés non phénoménologiques de la

21. Cf. H. G. Gadamer, op. cit., P. 135 : « "Konstitution" ist nichts anderes als "Wiederaufbaubewegung", die der vollzogenen Reduktion folgt. Wie die letztere transzendental ist, dh. keine reale Vernichtung meint, sondem nur die Suspension der Seinsgeltung, so ist auch der Aufbau aus den Leistungen der Subjektivität keine reale Erzeugung von ingend etwas, sondern der Weg des Verstehens all dessen, was als Sinn gelten soll. »

22. On pensera ici à la critique que Heidegger a faite de la phénoménologie husserlienne dans ses leçons de Marbourg et qui a déjà donné lieu à une très vaste littérature depuis l'étude de Giorgio Corà, « Ripetizione e superamento della fenomenologia in Martin Heidegger $\gg$, dans Verifiche 12, 1983, p. 371-409 ; 13, 1984, p. 1953. p. 281-316. En France, on pensera surtout aux travaux de J-L. Marion, Réduction et donation. Recherches sur Husserl, Heidegger et la phénoménologie, Paris, P.U.F., collection Epiméthée, 1989 et de J.-F. Courtine, Heidegger et la phénoménologie, Paris, Vrin, 1990. En Allemagne, cf. le recueil déjà cité Phänomenologie im Widerstreit. Tout se passe un peu comme si la publication des cours du jeune Heidegger rendait pour la première fois possible le débat que le dernier Husserl souhaitait conduire avec / contre Heidegger sur l'orientation ultime de la phénoménologie. 
phénoménologie, les orientant ainsi davantage vers la tâche d'une destruction de la métaphysique que vers celle d'un prolongement des avenues ouvertes par ses propres recherches.

Le tournant herméneutique (Heidegger, Gadamer) de la phénoménologie procède lui-même de tensions et d'ouvertures qui investissent déjà le projet husserlien. Elles ont trait à la conception de l'ego et à la conception transcendantale ou fondationnelle de la philosophie. Si l'ego qui intéresse la phénoménologie est d'emblée intentionnel, savoir un ego qui «s'éclate » dans des horizons de sens qui le dépassent, on peut se demander si l'ego peut encore être maintenu comme point de départ. Heidegger en a tiré la conclusion que l'ego était si peu présence à soi qu'il était d'abord et le plus souvent « être-dans-le-monde ». Dans son cours de 1927 sur les « problèmes fondamentaux de la phénoménologie », il a expressément présenté sa conception de l'être-aumonde comme une radicalisation de l'intentionnalité husserlienne, ce qu'il n'avait pas fait dans $\hat{E}$ tre et temps ${ }^{23}$. Mais la compréhension de l'intentionnalité à partir de l'horizon du monde, elle aussi préparée par Husserl, se double d'une métamorphose « éthique » de l'ego. Déchéant « sur » le monde, le sujet n'est pas d'abord auprès de soi, mais il a à le devenir. De prémisse, la présence à soi de l'ego, devenu Da-sein, devient en quelque sorte un impératif pratique chez Heidegger. S'abîmant dans des intentionnalités qui le dispersent aux quatre coins du monde, l'ego se manque d'abord lui-même. Heidegger brisait ainsi la conception théorique de l'ego qui gouvernait encore la phénoménologie de son maître. L'ego est d'abord un être de souci et dont le soin premier doit être celui d'un être-au-monde authentique. Cette réhabilitation de l'ego comme être de souci, comme projet pratique, n'est pas étrangère à la renaissance de la philosophie pratique qui s'est produite au sein même du mouvement phénoménologique, chez des auteurs aussi différents que H. Arendt, L. Strauss, J. P. Sartre, J. Patocka, E. Levinas, H. Jonas, G. Krüger, H. G. Gadamer ou H. Marcuse.

L'ego qui prend conscience de soi comme projet d'existence, comme souci de soi, se sait aussi projeté dans des horizons de sens qui le précèdent. L'idée herméneutique du Dasein comme

23. GA, t 24. p. 229-246 : « L'interprétation plus radicale de l'intentionnalité en vue d'une explication de la compréhension quotidienne de lêtre: lêtre-dans-le-monde comme fondement del'intentionnalité. » 
d'un projet jeté fait écho à la conception husserlienne du monde de la vie [Lebenswelt] comme source de toute constitution de sens. Husserl avait ainsi reconnu que l'ego n'était pas la source de tous ses projets de compréhension, reconduisant déjà ses horizons à l'anonymat - et, de plus en plus, à l'« intersubjectivité »-d'une Lebenswelt. On a longtemps pensé que la thématique de la Lebenswelt et de l'intersubjectivité n'était apparue que tardivement dans les préoccupations de Husserl et qu'elles trahissaient peut-être l'influence de Heidegger. Dans les œuvres publiées, l'intersubjectivité n'apparaissait effectivement que dans les Méditations cartésiennes et la Lebenswelt que dans la Krisis. C'était surestimer l'importance des publications pour Husserl! La publication posthume des Husserliana démontre que le thème de l'intersubjectivité ètait apparu beaucoup plus tôt (cf. notamment les tomes XIIIXIV) qu'on ne l'avait d'abord soupçonné. Cela est aussi vrai du concept de Iebenswelt, que le jeune Heidegger emploie déjà à profusion dans un cours de rgrg et sans prendre la peine de préciser

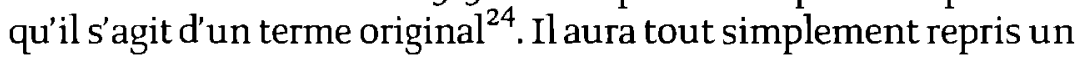
terme qu'il aura entendu de Husserl. L'idée même d'intentionnalité appelle le concept d'un monde de la vie qui devance la conscience de soi. C'était déjà reconnaître la finitude de l'ego.

On doit cependant se demander si cette conscience de la finitude ne finit pas par rendre problématique, sinon caduque, l'entreprise d'une philosophie comprise comme science apodictique et fondationnelle. Il semble que Husserl ait maintenu cette conception cartésienne de la philosophie jusqu'à la fin, même s'il n'a jamais prétendu l'avoir réalisée lui-même. Sa prétention était assurément plus modeste, comme en témoigne la Postface aux Ideen. Il tenait seulement à rappeler le souci de probité absolue que représente l'idée de la philosophie pour l'humanité. Mais une philosophie qui s'est avisée de l'éclatement constitutif de l'ego dans l'intentionnalité, de l'inscription de l'expérience du sens dans un monde vécu, peut-elle encore aspirer à une « fondation ultime » [Letztbegründungl, à un fundamentum inconcussum qui transcende l'histoire?

Ici, l'orientation herméneutique que Heidegger a proposée à la phénoménologie apparaît irrécusable et plus conforme aux

24. GA, Bd. 58 : Grundprobleme der Phänomenologie. Cours de $1919 / 1920$ paru en 1993. p. 54. 59 sq., 69, 76, 250, 26I et passim. Le terme apparaîtra souvent dans les cours suivants. 
choses elles-mêmes que ne l'était le « rêve» husserlien d'une fondation ultime : la sentence fameuse de la Krisis, « la philosophie comme science, comme science sérieuse, stricte, voire apodictiquece rêve est bel et bien fini $\gg^{25}$ vaut sans doute davantage pour les contemporains que pour Husserl lui-même, mais c'est bien lui qui a parlé d'un rêve ITrauml. Et s'il s'agissait après tout d'un rêve incompatible avec la finitude humaine? La contribution de Heidegger à la phénoménologie aura été de mettre à découvert les présupposés ontologiques de cette notion cartésienne de fondation ultime. La donnée première pour une phénoménologie herméneutique qui a radicalisé l'idée d'intentionnalité, au point de destituer l'ego de son rôle de sujet, est celle d'un Dasein fini, voué à des projets de sens dont le fondement dernier lui échappera toujours. Cherchant à fuir cette sienne temporalité, le Dasein " rêve », pour parler comme Husserl, d'un fundamentum inconcussum, mais ce rêve révèle surtout à quel point il est lui-même «concussum », temporel et fini ${ }^{26}$.

Dans le tournant herméneutique qu'il imprime à la phénoménologie, c'est l'idée même d'un fondement transcendantal (c'est-à-dire qui échappe tout à fait au temps) que Heidegger finit par mettre en question ${ }^{27}$. Il est clair que cette mise à distance de la notion de fondement, qui annonce le saut en amont de la raison (le Satz vom Grund de 1957), affole la conception classique de la philosophie et qu'elle soulève d'âpres problèmes. Elle est cependant libératrice en ceci qu'elle cesse d'orienter la connaissance philosophique sur un idéal qu'elle ne pourra jamais satisfaire. Cette phénoménologie devenue résolument herméneutique reste ainsi fidèle aux choses elles-mêmes, c'est-à-dire aux questions (ou

25. Husserliana VI, Beilage XXVIII du $\S 73$; tr. fr. par G. Granel, La crise des sciences européennes et la phénoménologie transcendantale, Paris, Gallimard, 1976, P. 563.

26. Sur cette temporalité radicale qui représente le point de départ ( « Être et temps ») de la phénoménologie heideggérienne, et sa véritable percée par rapport à Husserl, voir les premiers essais de notre ouvrage sur Lhorizon herméneutique de la pensée contemporaine, Paris, Vrin, 1993.

27. Cf. H. G. Gadamer, Wahrheit und Methode, dans l'édition de ses Gesammelte Werke, t. I, ig86. P. 261 (passage non traduit dans la traduction partielle de Vérité et méthode, Paris, Seuil, 1976) : « Il était donc clair que le projet d'une ontologie fondamentale chez Heidegger devait mettre le problème de lhistoire au premier plan. Néanmoins, il devint bientôt manifeste que ce nétait pas la solution du problème de lhistoricisme, ni une quelconque fondation plus originaire des sciences, ni même, comme chez Husserl, une autofondation radicale et ultime de la philosophie qui constituait le sens de cette ontologie fondamentale, mais que c'était plutôt l'idée même de fondation qui subissait un retournement complet. » 
intentions) les plus originaires de notre être. En tant qu'herméneutique de la facticité, elle s'est efforcée, avec Heidegger, d'éclaircir pour lui-même le Dasein, afin de lui rappeler qu'il était un projet jeté susceptible de prendre conscience de ses possibilités réelles dans l'ordre de l'histoire. Avec Gadamer, cette phénoménologie herméneutique s'est reconnue sous la logique de la question et de la réponse : tout énoncé doit être compris en fonction d'un dialogue plus originaire que lui. C'était une nouvelle fois mettre en question l'idéal husserlien d'un commencement absolu au nom de la poursuite d'un dialogue dans lequel il n'y a jamais de point zéro. La finitude, l'insertion de notre être dans des intentionnalités de sens qui l'excèdent, devient ainsi la donne première de la phénoménologie. Puisqu'elle nous concerne tous, elle est aussi universelle. Ainsi, l'herméneutique maintient la prétention à l'universalité, si chère à Husserl. C'est justement dans le renoncement à un fondement dernier que s'atteste son universalité. En ceci, l'herméneutique s'identifiera toujours à l'héritage de Husserl.

Université de Montréal 